\title{
THE CLASS OF FUNCTIONS SPIRALLIKE WITH RESPECT TO A BOUNDARY POINT
}

\author{
ADAM LECKO \\ Received 10 January 2003
}

\begin{abstract}
The aim of this paper is to present an analytic characterization of the class of functions $\delta$ spirallike with respect to a boundary point. The method of proof is based on Julia's lemma.

2000 Mathematics Subject Classification: 30C45.
\end{abstract}

1. Introduction. In this paper, we study the class $\mathscr{Y}_{0}^{*}(\delta)$ of $\delta$-spirallike functions with respect to a boundary point. Spirallikeness with respect to a boundary point is a fresh idea being the subject of studies in [1,2]. Cited papers developed the method based, on the one hand, on the analytic formula for the class $\mathscr{Y}_{0}^{*}$ of functions starlike with respect to a boundary point proposed and proved partially by Robertson [10], and, on the other hand, on some dynamical system built for $\mathscr{Y}_{0}^{*}(\delta)$. Lyzzaik [8] completing Robertson's proof solved positively his conjecture. Thereby the full analytic description of functions in $\mathscr{Y}_{0}^{*}$ was finished. The author [5], by using the Julia lemma, proposed an alternative analytic formula for the class $\mathscr{S}_{0}^{*}$ different than Robertson's characterization. The necessary condition for functions to be in $\mathscr{S}_{0}^{*}$ was shown and, partially, the sufficient condition. In [7], Lyzzaik and the author complete the proof and in this way the class $\mathscr{Y}_{0}^{*}$ was equipped with a new analytic characterization.

The use of the Julia lemma has the virtue of looking at the inner property of the class $\mathscr{Y}_{0}^{*}$ and the other classes defined by the geometric property connected with the boundary point (see, e.g., [6]). In this paper, we apply once again the Julia lemma as a technique to study the class $\mathscr{Y}_{0}^{*}(\delta)$. Theorem 3.5 demonstrates the basic observation that spirallikeness, as earlier starlikeness with respect to a boundary point, is preserved on each oricycle in the unit disk by every function in $\mathscr{Y}_{0}^{*}(\delta)$. Theorems 3.6 and 3.8 complete a new analytic characterization of $\delta$-spirallike functions with respect to a boundary point.

2. Preliminaries. Let $\mathbb{D}=\{z \in \mathbb{C}:|z|<1\}$ and let $\mathbb{T}=\partial \mathbb{D}$. For each $k>0$, consider the oricycle

$$
\mathbb{O}_{k}=\left\{z \in \mathbb{D}: \frac{|1-z|^{2}}{1-|z|^{2}}<k\right\} .
$$

The oricycle $\mathbb{O}_{k}$ is a disk in $\mathbb{D}$ whose boundary circle $\partial \mathbb{O}_{k}$ is tangent to $\mathbb{T}$ at 1 .

Let $\Delta=\{z \in \mathbb{D}:|\arg (1-z)|<b,|z-1|<\rho\}, b \in(0, \pi / 2), \rho<2 \cos b$, be a Stolz angle at 1 . 
Let $\mathscr{A}$ denote the set of all analytic functions in $\mathbb{D}$. The subset of $\mathscr{A}$ of all univalent functions will be denoted by $\mathscr{Y}$. The set of all $\omega \in \mathscr{A}$ such that $|\omega(z)|<1$ for $z \in \mathbb{D}$ will be denoted by $\mathscr{\Re}$.

An angular limit of $f \in \mathscr{A}$ at $\zeta \in \mathbb{T}$ will be denoted by $f_{L}(\zeta)$. An angular derivative of $f \in \mathscr{A}$ at $\zeta \in \mathbb{T}$ will be denoted by $f_{L}^{\prime}(\zeta)$.

Let $f \in \mathscr{A}$. Assume that there exists a finite radial $\operatorname{limit}_{r \rightarrow 1^{-}} f(r)=v$ at 1 . Denote by

$$
Q(z)=\frac{(z-1) f^{\prime}(z)}{f(z)-v}, \quad z \in \mathbb{D}
$$

the Visser-Ostrowski quotient of $f$ at 1 (see, e.g., [9, page 251]). We say that $f$ satisfies the Visser-Ostrowski condition at 1 if $Q_{<}(1)=1$ (see, e.g., [9, page 252]).

We recall now the Julia lemma (see [4]; see also [11, pages 68-72]).

Lemma 2.1 (Julia). Let $\omega \in \mathscr{B}$. Assume that there exists a sequence $\left(z_{n}\right)$ of points in $\mathbb{D}$ such that

$$
\begin{gathered}
\lim _{n \rightarrow \infty} z_{n}=1, \quad \lim _{n \rightarrow \infty} \omega\left(z_{n}\right)=1, \\
\lim _{n \rightarrow \infty} \frac{1-\left|\omega\left(z_{n}\right)\right|}{1-\left|z_{n}\right|}=\lambda<\infty .
\end{gathered}
$$

Then

$$
\frac{|1-\omega(z)|^{2}}{1-|\omega(z)|^{2}} \leq \lambda \frac{|1-z|^{2}}{1-|z|^{2}}, \quad z \in \mathbb{D},
$$

that is, for every $k>0$,

$$
\omega\left(\mathbb{O}_{k}\right) \subset \mathbb{O}_{\lambda k}
$$

REMARK 2.2. The constant $\lambda$ defined in (2.4) is positive (see [11, pages 68-69]).

For $\omega \in \mathscr{B}$ with $\omega_{\angle}(1)=1$, let

$$
\Lambda=\sup \left\{\frac{|1-\omega(z)|^{2}}{1-|\omega(z)|^{2}} \cdot \frac{1-|z|^{2}}{|1-z|^{2}}: z \in \mathbb{D}\right\} .
$$

The next lemma is a converse of the Julia lemma (see [11, page 72] and [3, pages 42-44]).

LEMMA 2.3. Let $\omega \in \mathscr{B}$. If (2.5) holds for some $\lambda>0$, then there exists a sequence $\left(z_{n}\right)$ of points in $\mathbb{D}$ satisfying (2.3) and such that

$$
\lim _{n \rightarrow \infty} \frac{1-\left|\omega\left(z_{n}\right)\right|}{1-\left|z_{n}\right|}=\Lambda \leq \lambda
$$


REMARK 2.4. In fact, in Lemma 2.3, we can find a sequence of real numbers $\left(x_{n}\right)$ in $(0,1)$ satisfying (2.3) and (2.8). Also it can be proved that then $\omega_{L}(1)=1$ and

$$
\lim _{\Delta \ni z \rightarrow 1} \frac{1-\omega(z)}{1-z}=\lim _{\Delta \ni z \rightarrow 1} \omega^{\prime}(z)=\omega_{L}^{\prime}(1)=\Lambda
$$

for every Stolz angle $\Delta$ (see [3, pages 42-44]).

For our need, it will be convenient to define the following classes of functions introduced in [5].

DEFINITION 2.5. Fix $\lambda \in(0, \infty]$. $\omega \in \mathscr{B}$ is said to belong to the class $\mathscr{B}(\lambda)$ if $\omega_{\angle}(1)=1$ and $\omega_{\angle}^{\prime}(1)=\lambda$.

Let $\mathscr{P}(\lambda)$ denote the class of all functions $p$ of the form

$$
p(z)=4 \frac{1-\omega(z)}{1+\omega(z)}, \quad z \in \mathbb{D}
$$

where $\omega \in \mathscr{B}(\lambda)$.

REMARK 2.6. Note that $p \in \mathscr{P}(\lambda)$ if and only if $p_{L}(1)=0$ and $p_{L}^{\prime}(1)=-2 \lambda$.

\section{Spirallikeness with respect to a boundary point.}

3.1. Let for $w \in \mathbb{C}$ and $A \subset \mathbb{C}, w A=\{w u: u \in A\}$.

We start with the following definition.

DeFinition 3.1. Fix $\delta \in(-\pi / 2, \pi / 2)$ and let $L(\delta)=\left\{\exp \left(e^{-i \delta} t\right): t \leq 0\right\}$ be the logarithmic spiral joint 0 and 1 . Clearly, $L(0)$ is a line segment $(0,1]$. Let $\mathscr{L}_{0}^{*}(\delta)$ denote the class of simply connected domains $\Omega \subset \mathbb{C}$ with $0 \in \partial \Omega$ and such that $w L(\delta) \subset \Omega$ for every $w \in \Omega$. Let $\mathscr{Y}_{0}^{*}(\delta) \subset \mathscr{Y}$ denote the corresponding class of functions mapping $\mathbb{D}$ onto domains in $\mathscr{L}_{0}^{*}(\delta)$.

Domains in $\mathscr{E}_{0}^{*}(\delta)$ and functions in $\mathscr{S}_{0}^{*}(\delta)$ will be called $\delta$-spirallike with respect to the boundary point at the origin.

For $\delta=0$, we get the class $\mathscr{Y}_{0}^{*}$, that is, $\mathscr{Y}_{0}^{*}=\mathscr{Y}_{0}^{*}(0)$. Recall that $f$ belongs to $\mathscr{Y}_{0}^{*}$ if and only if it is univalent in $\mathbb{D}$ and $f(\mathbb{D})$ is a starlike domain with respect to the boundary point at the origin, that is, the line segment $(0, w]$ is a subset of $f(\mathbb{D})$ for every $w \in f(\mathbb{D})$ (for more about the class $\mathscr{Y}_{0}^{*}$, see $[5,7,8,10]$ ).

Let $f \in \mathscr{Y}_{0}^{*}(\delta)$ for $\delta \in(-\pi / 2, \pi / 2)$, and fix $w_{1} \in f(\mathbb{D})$. Then $w_{1} L(\delta) \subset \Omega$ is a curve ending at the origin, so by [9, Proposition 2.1, page 29], the preimage of $w_{1} L(\delta)$ is a curve in $\mathbb{D}$ ending at some point $\zeta_{0}$ of $\mathbb{T}$. Applying [9, Corollary 2.17, page 35], we conclude that $f$ has the angular limit zero at $\zeta_{0}$.

Proposition 3.2. Every function $f \in \mathscr{Y}_{0}^{*}(\delta), \delta \in(-\pi / 2, \pi / 2)$, has the angular limit zero at some point $\zeta_{0} \in \mathbb{T}$, that is, $f_{L}\left(\zeta_{0}\right)=0$.

In the following considerations, we assume that $\zeta_{0}=1$, that is, we use the boundary normalization $f_{\angle}(1)=0$. 
3.2. In the proofs of the main theorems of this paper, we will need two lemmas proved in [5].

LEMMA 3.3. Every sequence $\left(a_{n}\right)$ of positive numbers with

$$
\lim _{n \rightarrow \infty}\left(a_{1} a_{2} \cdots a_{n}\right)=0
$$

has a convergent subsequence $\left(a_{n_{k}}\right)$ and

$$
0 \leq \lim _{k \rightarrow \infty} a_{n_{k}}=a \leq 1 .
$$

LEMMA 3.4. Let $f \in \mathscr{Y}$ have a radial limit $\lim _{r \rightarrow 1^{-}} f(r)=v$. Then there exist $\lambda \in[0,1]$ and a sequence $\left(r_{n}\right)$ with $0<r_{n}<1$ and $\lim _{n \rightarrow \infty} r_{n}=1$ such that

$$
\lim _{n \rightarrow \infty}\left|Q\left(r_{n}\right)\right|=2 \lambda
$$

3.3. The theorem below says that every function in $\mathscr{S}_{0}^{*}(\delta)$ having a boundary normalization $f_{\angle}(1)=0$ preserves spirallikeness with respect to a boundary point on each oricycle in $\mathbb{D}$. This information will be used later to find an analytic formula for functions in $\mathscr{S}_{0}^{*}(\delta)$.

THEOREM 3.5. Fix $\delta \in(-\pi / 2, \pi / 2)$ and let $f \in \mathscr{Y}$. Then $f \in \mathscr{Y}_{0}^{*}(\delta)$ and $f_{\angle}(1)=0$ if and only if $f\left(\mathbb{O}_{k}\right) \in \mathscr{L}_{0}^{*}(\delta)$ for every $k>0$.

Proof. Assume that $f \in \mathscr{S}_{0}^{*}(\delta)$ and $f_{L}(1)=0$. For each $t \leq 0$, define

$$
\omega_{t}(z)=f^{-1}\left(\exp \left(e^{-i \delta} t\right) f(z)\right), \quad z \in \mathbb{D} .
$$

Since $f(\mathbb{D}) \in \mathscr{L}_{0}^{*}(\delta)$, $\exp \left(e^{-i \delta} t\right) f(z) \in f(\mathbb{D})$ for every $t \leq 0, z \in \mathbb{D}$, and the univalence of $f$ shows that $\omega_{t}$ is well defined for each $t \leq 0$.

Now, fix $t<0$ and $w_{1} \in f(\mathbb{D})$. Hence $w_{1} L(\delta) \subset f(\mathbb{D})$. For $n \in \mathbb{N}$, let

$$
w_{n}=\exp \left(e^{-i \delta}(n-1) t\right) w_{1}
$$

and $z_{n}=f^{-1}\left(w_{n}\right)$. Since the sequence $\left(w_{n}\right)$ is placed on the logarithmic spiral $w_{1} L(\delta)$ and $\lim _{n \rightarrow \infty} w_{n}=0, \lim _{n \rightarrow \infty} z_{n}=1$ by Proposition 3.2. Observe that

$$
\omega_{t}\left(z_{n}\right)=f^{-1}\left(\exp \left(e^{-i \delta} t\right) w_{n}\right)=f^{-1}\left(\exp \left(e^{-i \delta} n t\right) w_{1}\right)=z_{n+1} .
$$

Let now

$$
a_{n}=\frac{1-\left|\omega_{t}\left(z_{n}\right)\right|}{1-\left|z_{n}\right|}, \quad n \in \mathbb{N} \text {. }
$$

Hence

$$
a_{n}=\frac{1-\left|z_{n+1}\right|}{1-\left|z_{n}\right|}
$$


for all $n \in \mathbb{N}$. Consequently,

$$
\begin{aligned}
\lim _{n \rightarrow \infty}\left(a_{1} a_{2} \cdots a_{n}\right) & =\lim _{n \rightarrow \infty}\left(\frac{1-\left|z_{2}\right|}{1-\left|z_{1}\right|} \frac{1-\left|z_{3}\right|}{1-\left|z_{2}\right|} \cdots \frac{1-\left|z_{n}\right|}{1-\left|z_{n-1}\right|} \frac{1-\left|z_{n+1}\right|}{1-\left|z_{n}\right|}\right) \\
& =\lim _{n \rightarrow \infty} \frac{1-\left|z_{n+1}\right|}{1-\left|z_{1}\right|}=0 .
\end{aligned}
$$

Lemma 3.3 yields a convergent subsequence $\left(a_{n_{k}}\right)$ such that

$$
0 \leq \lim _{k \rightarrow \infty} a_{n_{k}}=\lambda(t) \leq 1
$$

which means that

$$
\lim _{k \rightarrow \infty} \frac{1-\left|\omega_{t}\left(z_{n_{k}}\right)\right|}{1-\left|z_{n_{k}}\right|}=\lambda(t) \leq 1
$$

for each $t<0$. In view of Remark 2.2, $\lambda(t)>0$ for every $t<0$.

Hence, each $\omega_{t}$ satisfies the assumptions of the Julia lemma, and since $\lambda(t) \leq 1$ for every $t<0$, we derive that $\omega_{t}\left(\mathbb{O}_{k}\right) \subset \mathbb{O}_{\lambda(t) k} \subset \mathbb{O}_{k}$ for every $k>0$. This yields $\exp \left(e^{-i \delta} t\right) f\left(\mathbb{O}_{k}\right) \subset f\left(\mathbb{O}_{k}\right)$ for every $t<0$, and hence $f\left(\mathbb{O}_{k}\right) \in \mathscr{L}_{0}^{*}(\delta)$.

Conversely, assume that $f\left(\mathbb{O}_{k}\right) \in \mathscr{L}_{0}^{*}(\delta)$ for every $k>0$. Since $0 \in \bigcap_{k>0} \partial f\left(\mathbb{O}_{k}\right)$ and

$$
f(\mathbb{D})=\bigcup_{k>0} f\left(\mathbb{O}_{k}\right),
$$

it follows that $0 \in \partial f(\mathbb{D})$ and $f(\mathbb{D}) \in \mathscr{L}_{0}^{*}(\Delta)$, so $f \in \mathscr{Y}_{0}^{*}(\delta)$. We show that $f_{\angle}(1)=0$. Fix $k>0$ and $w_{1} \in f\left(\mathbb{O}_{k}\right)$. Then $w_{1} L(\delta) \subset f\left(\mathbb{O}_{k}\right)$ is a curve ending at $0 \in \partial f(\mathbb{D})$. By [9, Proposition 2.14, page 29], $f^{-1}\left(w_{1} L(\delta)\right)$ is a curve in $\mathbb{D}$ ending at some point $\zeta_{0}$ of $\mathbb{T}$. Since $f^{-1}\left(w_{1} L(\delta)\right) \subset \mathbb{O}_{k}$ and $\overline{\mathbb{O}_{k}} \cap \mathbb{T}=\{1\}$, we have $\zeta_{0}=1$. The proof of the theorem is finished.

Using Theorem 3.5, we characterize functions in $\mathscr{Y}_{0}^{*}(\delta)$ as follows.

THEOREM 3.6. Fix $\delta \in(-\pi / 2, \pi / 2)$. If $f \in \mathscr{P}_{0}^{*}(\delta)$ and $f_{L}(1)=0$, then there exist $\lambda \in(0,1]$ and $\omega \in \mathscr{B}(\lambda)$ such that

$$
-e^{i \delta}(1-z)^{2} \frac{f^{\prime}(z)}{f(z)}=4 \frac{1-\omega(z)}{1+\omega(z)}, \quad z \in \mathbb{D} .
$$

Proof. The case $\delta=0$ reduces to [5, Theorem 3.1]. Therefore we assume that $\delta \in$ $(-\pi / 2, \pi / 2) \backslash\{0\}$. Let $O_{k}=\partial \mathbb{O}_{k} \backslash\{1\}$ and $\Gamma_{k}=\partial f\left(\mathbb{O}_{k}\right)$ for every $k>0$. First, we show that

$$
\operatorname{Re}\left\{e^{i \delta}(1-z)^{2} \frac{f^{\prime}(z)}{f(z)}\right\}<0, \quad z \in \mathbb{D} .
$$

We prove that the last inequality is true for all points on $O_{k}$ for every $k>0$. Now, fix $k>0, z \in O_{k}$ and let $w=f(z)$. We parametrize $O_{k}$ as follows:

$$
O_{k}: z=\gamma_{k}(\theta)=\frac{1+k e^{i \theta}}{1+k}, \quad \theta \in(0,2 \pi) .
$$


Thus $O_{k}$ is positively oriented. Denote by $\tau(z)$ the tangent vector to $\Gamma_{k}$ at $w=f(z)$, that is, $\tau(z)=\gamma_{k}^{\prime}(\theta) f^{\prime}\left(\gamma_{k}(\theta)\right)$, where $z=\gamma_{k}(\theta)$. Since

$$
\begin{aligned}
\left(1-\gamma_{k}(\theta)\right)^{2} & =\frac{k^{2}}{(1+k)^{2}}\left(1-e^{i \theta}\right)^{2}=-\frac{4 k \sin ^{2}(\theta / 2)}{(k+1) i}\left(\frac{k}{k+1} e^{i \theta} i\right) \\
& =\frac{4 k \sin ^{2}(\theta / 2)}{k+1} \gamma_{k}^{\prime}(\theta) i=2 \operatorname{Re}\left\{1-\gamma_{k}(\theta)\right\} \gamma_{k}^{\prime}(\theta) i, \quad \theta \in(0,2 \pi),
\end{aligned}
$$

we have

$$
\tau(z)=\frac{-i\left(1-\gamma_{k}(\theta)\right)^{2} f^{\prime}\left(\gamma_{k}(\theta)\right)}{2 \operatorname{Re}\left\{1-\gamma_{k}(\theta)\right\}}=\frac{-i(1-z)^{2} f^{\prime}(z)}{2 \operatorname{Re}\{1-z\}} .
$$

Let

$$
w(t)=f(z) \exp \left(e^{-i \delta} t\right), \quad t \leq 0
$$

be a parametrization of $f(z) L(\delta)$ and let $w^{\prime}(0)=\lim _{t \rightarrow 0^{-}} w^{\prime}(t)=e^{-i \delta} f(z)$ be the onesided tangent vector to the logarithmic spiral $f(z) L(\delta)$ at $f(z)$. By $\varphi(z)$ we denote the directed angle from the vector $i w^{\prime}(0)$ to $\tau(z)$, that is,

$$
\begin{aligned}
\varphi(z) & =\arg \{\boldsymbol{\tau}(z)\}-\arg \left\{i w^{\prime}(0)\right\} \\
& =\arg \left\{\frac{-i(1-z)^{2} f^{\prime}(z)}{2 \operatorname{Re}\{1-z\}}\right\}-\arg \left\{i e^{-i \delta} f(z)\right\} \\
& =\arg \left\{-e^{i \delta} \frac{(1-z)^{2} f^{\prime}(z)}{f(z)}\right\} .
\end{aligned}
$$

By Theorem 3.5, $f\left(\mathbb{O}_{k}\right) \in \mathscr{L}_{0}^{*}(\delta)$ for every $k>0$. Hence it is easy to see that

$$
w L(\delta) \subset \overline{f\left(\mathbb{O}_{k}\right)},
$$

where $w=f(z) \in \Gamma_{k}$. Indeed, let $w_{0} \in w L(\delta)$ be arbitrary. Thus $w_{0}=w u_{0}$ for some $u_{0} \in L(\delta)$. Since $w \in \Gamma_{k}$, there exists a sequence $\left(w_{n}\right)$ of points in $f\left(\mathbb{O}_{k}\right)$ convergent to $w$. The inclusion $w_{n} L(\delta) \subset f\left(\mathbb{O}_{k}\right)$ yields that $w_{n} u_{0}$ is a point of $f\left(\mathbb{O}_{k}\right)$ for every $n \in \mathbb{N}$. At the end, the convergence of the sequence $\left(w_{n} u_{0}\right)$ of points of $f\left(\mathbb{O}_{k}\right)$ to $w_{0}$ implies that $w_{0} \in \overline{f\left(\mathbb{O}_{k}\right)}$. Since $w_{0}$ was arbitrary, our claim is proved.

Let $l$ be a line going through $f(z)$ with $w^{\prime}(0)$ as the directional vector. Then $l$ divides the plane into two closed half-planes $H_{1}$ and $H_{2}$. One of them, say $H_{1}$, contains the origin and the spiral $f(z) L(\delta)$. We assume first that $\delta \in(-\pi / 2,0)$. This means that the spiral $L(\delta)$ has the shape such that it attains 1 from the lower half-plane. Moreover, $f(z) L(\delta)$ parametrized as above turns round the origin in the counterclockwise direction. Hence, $i w^{\prime}(0)$ lies in $H_{1}$. By Theorem 3.5, $f\left(\mathbb{O}_{k}\right) \in \mathscr{L}_{0}^{*}(\delta)$. Hence, and from (3.20), it follows that either $\Gamma_{k}$ is tangent both to $f(z) L(\delta)$ (one-sided) and to $l$ at $f(z)$, and then $\tau(z)$ lies in $l$ so in $H_{1}$, or by [9, Proposition 2.13, page 28], there is a crosscut $C \subset l$ of $f\left(\mathbb{O}_{k}\right)$ with one endpoint at $f(z)$. Thus, by [9, Proposition 2.12, page 27], $f\left(\mathbb{O}_{k}\right)$ has exactly two components, one of them, say $G$, lies in $H_{2}$. Clearly, $\partial G=C \cup \Gamma$, where $\Gamma \subset \Gamma_{k}$ ends at $f(z)$. Hence $\Gamma$ is a subset of $H_{2}$ and, since it is part of a positively oriented closed 
analytic curve $\Gamma_{k}$, we deduce finally that the tangent vector $\tau(z)$ to $\Gamma_{k}$ at $f(z)$ lies in $H_{1}$. In a similar way, we can prove that both vectors $i w^{\prime}(0)$ and $\tau(z)$ lie together in $H_{2}$ as $\delta \in(0, \pi / 2)$. This, (3.19), and the fact that $i w^{\prime}(0)$ is orthogonal to $l$ yield

$$
|\varphi(z)| \leq \frac{\pi}{2}
$$

As $k>0$ and $z \in O_{k}$ was arbitrary, this is true in $\mathbb{D}$.

Suppose now that equality holds in (3.21) for some $z_{0} \in \mathbb{D}$. By the maximum principle for harmonic functions, it holds in the whole disk $\mathbb{D}$, which implies that there exists $y \in \mathbb{R} \backslash\{0\}$ so that

$$
e^{i \delta}(1-z)^{2} \frac{f^{\prime}(z)}{f(z)} \equiv y i, \quad z \in \mathbb{D} .
$$

But the solution

$$
f(z)=f_{0}(z)=f(0) \exp \left(\frac{e^{-i \delta} y i z}{1-z}\right), \quad z \in \mathbb{D},
$$

of the last equation is not univalent in $\mathbb{D}$. So $f_{0} \notin \mathscr{S}_{0}^{*}(\delta)$, and hence strict inequality holds in (3.21).

Let $p(z)=-e^{i \delta}(1-z)^{2} f^{\prime}(z) / f(z)$ and let

$$
\omega(z)=\frac{4-p(z)}{4+p(z)}, \quad z \in \mathbb{D} .
$$

Then $\omega(\mathbb{D}) \subset \mathbb{D}$. We now prove that $\omega \in \mathscr{B}(\lambda)$ for some $\lambda \in(0,1]$. Recalling the VisserOstrowski quotient, we can write

$$
p(z)=e^{i \delta}(1-z) Q(z), \quad z \in \mathbb{D} .
$$

Since, for every $r \in(0,1)$,

$$
|Q(r)| \leq \frac{4}{1+r}
$$

(see [5, Lemma 2.2, (2.3)]), we have

$$
\lim _{r \rightarrow 1^{-}}\{(1-r) Q(r)\}=\lim _{r \rightarrow 1^{-}}\left\{e^{-i \delta} p(r)\right\}=0 .
$$

Hence $\lim _{r \rightarrow 1^{-}} p(r)=0$ and, in view of (3.24), $\lim _{r \rightarrow 1^{-}} \omega(r)=1$, so condition (2.3) of the Julia lemma is satisfied. By Lemma 3.4, there exist $\lambda_{0} \in[0,1]$ and a sequence $\left(r_{n}\right)$ in $(0,1)$ with $\lim _{n \rightarrow \infty} r_{n}=1$ such that

$$
\lim _{n \rightarrow \infty}\left|Q\left(r_{n}\right)\right|=2 \lambda_{0} .
$$


From (3.24) and (3.27) we have

$$
\begin{aligned}
\lim _{n \rightarrow \infty} \frac{\left|1-\omega\left(r_{n}\right)\right|}{1-r_{n}} & =\lim _{n \rightarrow \infty}\left\{\frac{2}{\left|4+p\left(r_{n}\right)\right|} \cdot \frac{\left|p\left(r_{n}\right)\right|}{1-r_{n}}\right\} \\
& =\lim _{n \rightarrow \infty}\left\{\frac{2}{\left|4+p\left(r_{n}\right)\right|}\left|Q\left(r_{n}\right)\right|\right\} \\
& =\lambda_{0} \in[0,1] .
\end{aligned}
$$

But

$$
\frac{1-\left|\omega\left(r_{n}\right)\right|}{1-r_{n}} \leq \frac{\left|1-\omega\left(r_{n}\right)\right|}{1-r_{n}},
$$

so we can find a subsequence $\left(r_{n_{k}}\right)$ of $\left(r_{n}\right)$ such that

$$
\lim _{k \rightarrow \infty} \frac{1-\left|\omega\left(r_{n_{k}}\right)\right|}{1-r_{n_{k}}}=\lambda_{1} \leq \lambda_{0} .
$$

By Remark 2.2, $\lambda_{1} \in(0,1]$. Hence $\omega$ satisfies the assumptions of the Julia lemma with $\lambda=\lambda_{1}$. Since then (2.5) holds, by using Lemma 2.3 and Remark 2.4 , we see that $\omega \in$ $\mathscr{B}(\Lambda)$, where $\Lambda \leq \lambda_{1} \leq 1$ is given by (2.7). This ends the proof of the theorem.

COROLlary 3.7. If $f \in \mathscr{Y}_{0}^{*}(\delta), \delta \in(-\pi / 2, \pi / 2)$, and $f_{L}(1)=0$, then there exists $\lambda \in(0,1]$ such that

$$
\lim _{\Delta \ni z \rightarrow 1} Q(z)=2 \lambda e^{-i \delta}
$$

for every Stolz angle $\Delta$.

Proof. Since

$$
\begin{aligned}
Q(z)= & e^{-i \delta} \frac{4}{1+\omega(z)} \frac{1-\omega(z)}{1-z}, \quad z \in \mathbb{D}, \\
& \lim _{\Delta \ni z \rightarrow 1} \frac{1-\omega(z)}{1-z}=\Lambda \in(0,1]
\end{aligned}
$$

for every Stolz angle $\Delta$, the assertion follows at once with $\lambda=\Lambda$.

TheOREM 3.8. Fix $\delta \in(-\pi / 2, \pi / 2)$. Let $f \in \mathscr{A}$ with $f_{L}(1)=0$. If there exist $\lambda \in$ $\left(0, \cos \delta\right.$ ] and a function $\omega \in \mathscr{B}(\lambda)$ such that (3.13) holds, then $f \in \mathscr{Y}_{0}^{*}(\delta)$.

Proof. First we show that $f$ is univalent in $\mathbb{D}$. It is immediate from (3.13) that $f$ is locally univalent in $\mathbb{D}$. Let $\omega \in \mathscr{B}(\lambda)$, where $\lambda \in(0, \cos \delta]$, and let $g$ be the solution of the differential equation

$$
-(1-z)^{2} \frac{g^{\prime}(z)}{g(z)}=4 \frac{1-\omega(z)}{1+\omega(z)}, \quad z \in \mathbb{D}
$$

with the boundary condition $g_{\llcorner}(1)=0$. As was proved in [7, Theorem 3], $g$ belongs to the class $\mathscr{Y}_{0}^{*}$, so it is univalent, and $g(\mathbb{D})$ being a simply connected domain lies in a 
wedge of angle $2 \lambda \pi$. Hence there exists a single-valued analytic branch of $\log g$ in $\mathbb{D}$, and

$$
g^{e^{-i \delta}}(z)=\exp \left\{e^{-i \delta} \log g(z)\right\}, \quad z \in \mathbb{D},
$$

is well defined. But, in view of (3.13) and (3.34), we have

$$
\frac{g^{\prime}}{g}=e^{i \delta} \frac{f^{\prime}}{f}
$$

so

$$
f=g^{e^{-i \delta}}
$$

Since $\lambda \in(0, \cos \delta]$, from the above, the univalence of $f$ in $\mathbb{D}$ follows.

Now, we prove that $f(\mathbb{D}) \in \mathscr{L}_{0}^{*}(\delta)$. This is clear, looking at the relation (3.37) between classes $\mathscr{S}_{0}^{*}$ and $\mathscr{S}_{0}^{*}(\delta)$, which yields the geometric relation between starlikeness and spirallikeness of domains in the plane. To be self-contained, we prove it without using geometric properties of functions in $\mathscr{Y}_{0}^{*}$. We assume that $\delta \neq 0$, since this case reduces to [7, Theorem 3].

Let $O_{k}=\partial \mathbb{O}_{k} \backslash\{1\}$ and $\Gamma_{k}=\partial f\left(\mathbb{O}_{k}\right)$ for every $k>0$. Suppose, on the contrary, that $f(\mathbb{D}) \notin \mathscr{E}_{0}^{*}(\delta)$. By Theorem 3.5, there exists $k>0$ such that $f\left(\mathbb{O}_{k}\right) \notin \mathscr{L}_{0}^{*}(\delta)$. Hence $w_{0} L(\delta) \not \subset f\left(\mathbb{O}_{k}\right)$ for some $w_{0} \in f\left(\mathbb{O}_{k}\right)$. Thus there exists $w_{1} \in\left(w_{0} L(\delta) \backslash\left\{w_{0}\right\}\right) \cap \Gamma_{k}$ such that the subarc of $w_{0} L(\delta)$ joining $w_{1}$ and $w_{0}$ without $w_{1}$ is contained in $f\left(\mathbb{O}_{k}\right)$. Since $w_{1} \in \Gamma_{k}, w_{1}=f\left(z_{1}\right)$ for some $z_{1} \in O_{k}$. Let

$$
v(t)=w_{0} \exp \left\{e^{-i \delta} t\right\}, \quad t \leq 0
$$

be a parametrization of $w_{0} L(\delta)$. Clearly,

$$
w_{1}=v\left(t_{1}\right)=w_{0} \exp \left\{e^{-i \delta} t_{1}\right\}
$$

for some $t_{1}<0$. Let

$$
w(t)=w_{1} \exp \left\{e^{-i \delta} s\right\}, \quad s \leq 0,
$$

be a parametrization of $w_{1} L(\delta)$. From (3.38), (3.39), and (3.40), we have

$$
w(t)=w_{0} \exp \left\{e^{-i \delta} t_{1}\right\} \exp \left\{e^{-i \delta} s\right\}=w_{0} \exp \left\{e^{-i \delta}\left(t_{1}+s\right)\right\},
$$

which means that $w_{1} L(\delta)$ is a subset of $w_{0} L(\delta)$. Moreover,

$$
v^{\prime}\left(t_{1}\right)=e^{-i \delta} w_{0} \exp \left\{e^{-i \delta} t_{1}\right\}=e^{-i \delta} f\left(z_{1}\right)=w^{\prime}(0) .
$$

Therefore the tangent line $l$ to $w_{0} L(\delta)$ at $w_{1}$ has the directional vector $v^{\prime}\left(t_{1}\right)=w^{\prime}(0)$ and is the boundary of two closed half-planes denoted by $H_{1}$ and $H_{2}$. One of them, say $H_{1}$, contains the origin. Let $\delta \in(-\pi / 2,0)$. As we remarked in the proof of Theorem 3.6, 
the spiral $L(\delta)$ has the shape such that it attains 1 from the lower half-plane. Moreover, $w_{1} L(\delta)$ parametrized as above turns round the origin in the counterclockwise direction. Hence, $i w^{\prime}(0)$ lies in $H_{1}$. Observe that either $\Gamma_{k}$ is tangent as well to $w_{1} L(\delta)$ (one-sided) as to $l$ at $w_{1}$ and then $\tau\left(z_{1}\right)$ lies in $l$, or, by [9, Proposition 2.13, page 28], there is a crosscut $C \subset l$ of $f\left(\mathbb{O}_{k}\right)$ with one endpoint at $w_{1}$. Thus, by [9, Proposition 2.12, page 27], $f\left(\mathbb{O}_{k}\right)$ has exactly two components, one of them, say $G$, lies in $H_{2}$. Moreover, $\partial G=C \cup \Gamma$, where $\Gamma \subset \Gamma_{k}$ ends at $w_{1}$. Hence $\Gamma$ is a subset of $H_{2}$ and, since it is part of a positively oriented closed analytic curve $\Gamma_{k}$, we deduce finally that the tangent vector $\tau\left(z_{1}\right)$ to $\Gamma_{k}$ at $f\left(z_{1}\right)$ lies in $H_{2}$. Since $i w^{\prime}(0)$ is orthogonal to $l$ and lies in $H_{1}$, we deduce that

$$
\left|\varphi\left(z_{1}\right)\right| \geq \frac{\pi}{2}
$$

where $\varphi\left(z_{1}\right)$ denotes the directed angle defined by (3.19), with $z_{1}$ instead of $z$. This contradicts (3.13). Similarly, we get a contradiction assuming that $\delta \in(0, \pi / 2)$.

REMARK 3.9. In [1], the authors found necessary and sufficient conditions for functions to be in $\mathscr{Y}_{0}^{*}(\delta)$ (Theorem 2.1). The analytic formula (2.1) in [1] generalizes the Robertson inequality for starlike functions with respect to a boundary point. In fact, the authors of [1] proved that each spirallike function with respect to a boundary point is a complex power of a corresponding function which is starlike with respect to a boundary point. Formula (3.13) presents an alternative analytic description of the class $\mathscr{Y}_{0}^{*}(\delta)$. In case $\delta=0(\mu=2 \pi$ in [1, equation (2.1)]), these two analytic formulas for $\mathscr{S}_{0}^{*}(0)$ characterizing starlike functions with respect to a boundary point are equivalent. Looking at [1, Theorem 2.1(III) and Theorems 3.2 and 3.3], we can expect that formulas (2.1) in [1] and (3.13) of the present paper are equivalent, which, in fact, means that in Theorem 3.6 the assumptions $\lambda \in(0,1]$ should be replaced by $\lambda \in(0, \cos \delta]$. This is an open problem.

3.4. Now, we present some examples of functions. In all of the examples below, $\delta \in(-\pi / 2, \pi / 2)$ and $p(z)=-e^{i \delta}(1-z)^{2} f^{\prime}(z) / f(z)$. It is convenient to express formula (3.13) in terms of the class $\mathscr{P}(\lambda)$. Therefore, in the examples below, we apply Remark 2.6 which says that $p \in \mathscr{P}(\lambda)$ if and only if $p_{L}(1)=0$ and $p_{L}^{\prime}(1)=-2 \lambda$. In every case, we use Theorem 3.8 reformulated by using the class $\mathscr{P}(\lambda)$.

EXAMPLE 3.10. (1) $f(z)=((1-z) /(1+z))^{\beta e^{-i \delta}}, \beta>0, z \in \mathbb{D}$.

Then $p(z)=2 \beta(1-z) /(1+z)$. Hence $\operatorname{Re} p(z)>0, z \in \mathbb{D}, p(1)=0$, and $p^{\prime}(1)=-\beta$. Consequently, $f \in \mathscr{Y}_{0}^{*}(\delta)$ for $\beta \in(0,2]$. For every $\beta>2, f \notin \mathscr{Y}_{0}^{*}(\delta)$.

(2) $f(z)=(1-z)^{\beta e^{-i \delta}}, \beta>0, z \in \mathbb{D}$.

Then $p(z)=\beta(1-z)$. Hence $\operatorname{Re} p(z)>0, z \in \mathbb{D}, p(1)=0$, and $p^{\prime}(1)=-\beta$. Consequently, $f \in \mathscr{Y}_{0}^{*}(\delta)$ for $\beta \in(0,2]$. For every $\beta>2, f \notin \mathscr{Y}_{0}^{*}(\delta)$.

(3) $f(z)=(1-z)^{2 e^{-i \delta}} e^{e^{-i \delta} z}, z \in \mathbb{D}$.

Then $p(z)=-z^{2}+1$. Hence $\operatorname{Re} p(z)>0, z \in \mathbb{D}, p(1)=0$, and $p^{\prime}(1)=-2$. Consequently, $f \in \mathscr{S}_{0}^{*}(\delta)$.

ACKNOwLEDGMENT. The author would like to thank prof. D. Shoikhet who, during the conference in honour of prof. L. Zalcman organized by Braude College in Israel in 2003, pointed out an error in the proof of Theorem 3.8. 


\section{REFERENCES}

[1] D. Aharonov, M. Elin, and D. Shoikhet, Spiral-like functions with respect to a boundary point, J. Math. Anal. Appl. 280 (2003), no. 1, 17-29.

[2] M. Elin, S. Reich, and D. Shoikhet, Holomorphically accretive mappings and spiral-shaped functions of proper contractions, Nonlinear Anal. Forum 5 (2000), 149-161.

[3] J. B. Garnett, Bounded Analytic Functions, Pure and Applied Mathematics, vol. 96, Academic Press, New York, 1981.

[4] G. Julia, Extension nouvelle d'un lemme de Schwarz, Acta Math. 42 (1920), 349-355 (French).

[5] A. Lecko, On the class of functions starlike with respect to a boundary point, J. Math. Anal. Appl. 261 (2001), no. 2, 649-664.

[6] - On the class of functions convex in the negative direction of the imaginary axis, J. Aust. Math. Soc. 73 (2002), no. 1, 1-10.

[7] A. Lecko and A. Lyzzaik, A note on univalent functions starlike with respect to a boundary point, J. Math. Anal. Appl. 282 (2003), no. 2, 846-851.

[8] A. Lyzzaik, On a conjecture of M. S. Robertson, Proc. Amer. Math. Soc. 91 (1984), no. 1, 108-110.

[9] Ch. Pommerenke, Boundary Behaviour of Conformal Maps, Grundlehren der mathematischen Wissenschaften, vol. 299, Springer-Verlag, Berlin, 1992.

[10] M. S. Robertson, Univalent functions starlike with respect to a boundary point, J. Math. Anal. Appl. 81 (1981), no. 2, 327-345.

[11] G. Sansone and J. Gerretsen, Lectures on the Theory of Functions of a Complex Variable. II: Geometric Theory, Wolters-Noordhoff Publishing, Groningen, 1969.

Adam Lecko: Department of Mathematics, Rzeszów University of Technology, Wincentego Pola 2, 35-959 Rzeszów, Poland

E-mail address: a1ecko@prz.rzeszow.p1 


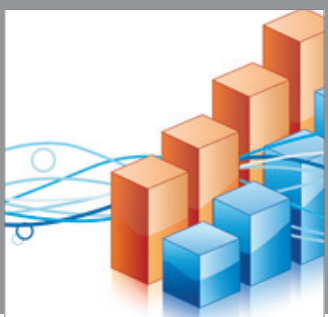

Advances in

Operations Research

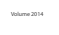

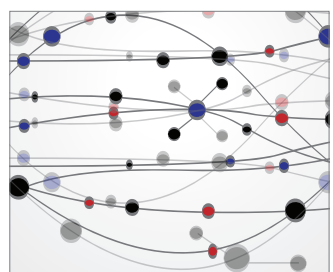

\section{The Scientific} World Journal
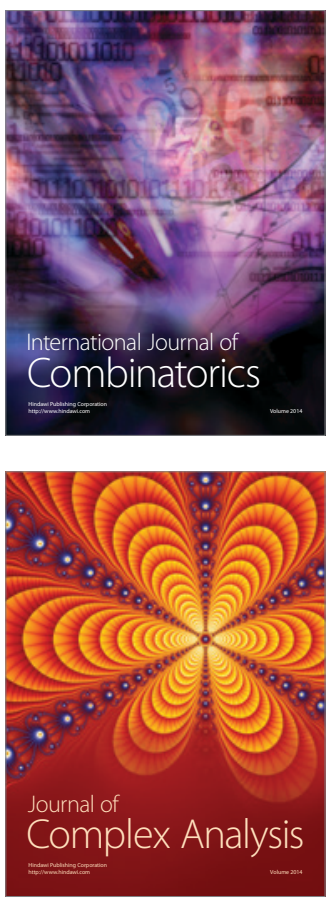

International Journal of

Mathematics and

Mathematical

Sciences
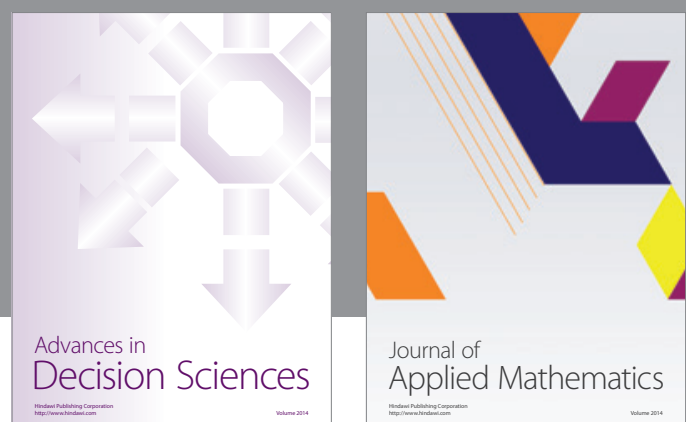

Journal of

Applied Mathematics
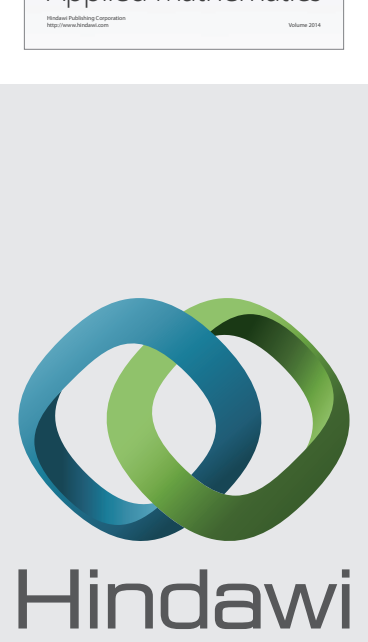

Submit your manuscripts at http://www.hindawi.com
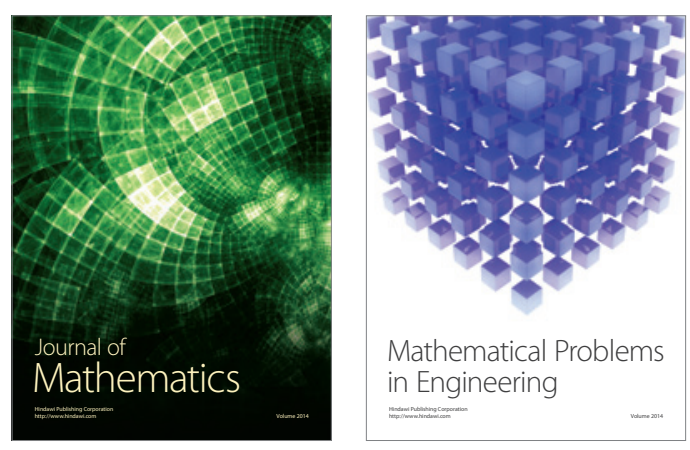

Mathematical Problems in Engineering
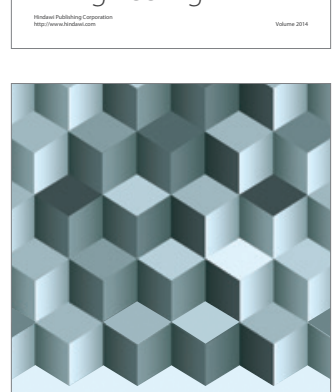

Journal of

Function Spaces
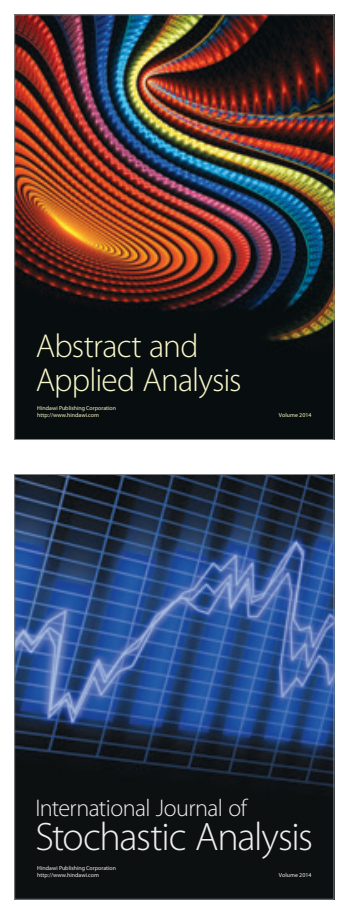

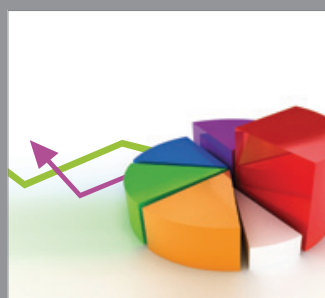

ournal of

Probability and Statistics

Promensencen
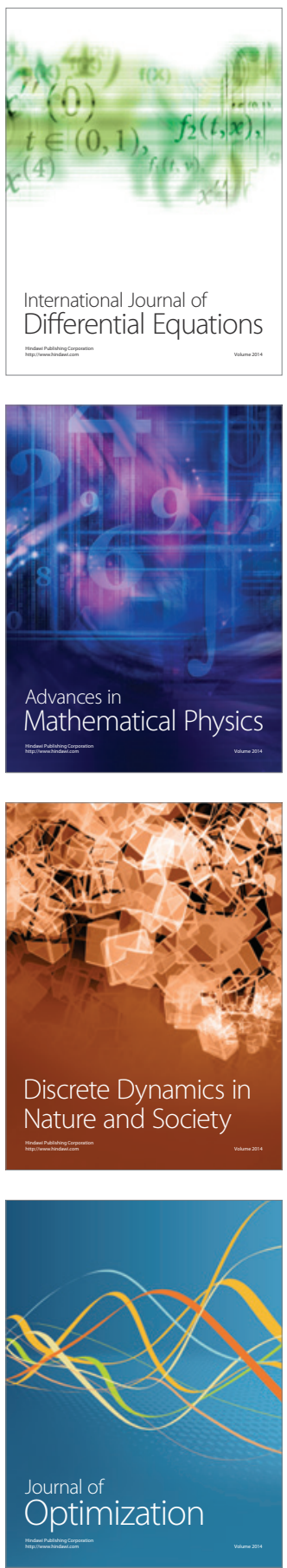